\title{
Numerical simulation of coupled problems
}

\author{
A. Harapin, J. Radnić \& M. Sunara \\ Faculty of Civil Engineering, Architecture and Geodesy, \\ University of Split, Croatia
}

\begin{abstract}
The paper briefly describes one numerical model for the simulation of fluidstructure coupled problems. The presented model is primarily intended to simulate the fluid-structure dynamic interaction in seismic conditions of civil engineering structures which are in direct contact with fluid and which can often be encountered in engineering practice, for example: dams, water tanks (reservoirs), offshore structures, pipelines, water towers, etc. The model is based on the so called "partition scheme" where the equations governing the fluid's pressures and the displacement of the structure are solved separately, with two distinct solvers. The SPH (Smooth Particle Hydrodynamics) method is used for the fluid and the standard FEM (Finite Element Method) is used for the structure, which can be made from reinforced concrete or steel and which can be simulated with shell or 3D elements. The model includes the most important nonlinear effects of reinforced concrete behaviour: yielding in compression and opening and propagation of cracks in tension (with tensile and shear stiffness of cracked concrete), as well as steel behaviour: yielding in compression and tension. The most important nonlinear effects of the fluid can also be simulated, like fluid viscosity, turbulence and cavitation. Some of the model's possibilities are illustrated in a practical example.

Keywords: coupled problems, fluid-structure interaction, SPH method, nonlinear behaviour, shell structures, water impact.
\end{abstract}

\section{Introduction}

Structures which are in direct contact with fluid, for example: dams, water tanks (reservoirs), off shore structures, pipelines, water towers etc., are very common in engineering practice. Numerical models for realistic simulations of these structures have to include the simulation of the fluid-structure interaction to 
ascertain the real behaviour of this complex system. This problem is particularly emphasized under dynamic (seismic) conditions and it is commonly referred to as a Fluid-structure interaction (or a coupled, or a multi-field) problem, Hou et al. [1]. Some of those structures can be seen in fig. 1 .

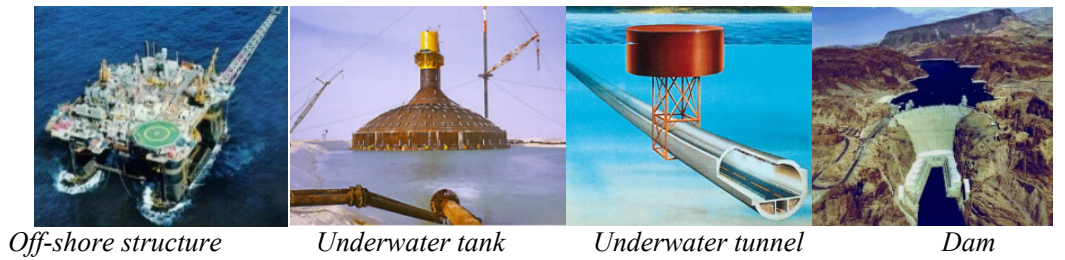

Figure 1: Examples of structures in direct contact with fluid.

Fluid-structure interaction (FSI) problems in general, are problems where solid structures interact with internal or surrounding fluid. FSI problems can be encountered in many scientific and engineering fields. Aside from in civil engineering, they can be found in naval engineering (ship stability, propeller blade design...), mechanical engineering (design of turbines, pipes...), aerodynamics (design of aircraft wings...), biomechanics (blood-heart interaction, inner ear fluid dynamics, jellyfish swimming, sperm motility, etc.), electro-hydrodynamics, magneto-hydrodynamic flows, etc.

But in spite of the very hard work of many scientists, a comprehensive study of such problems remains a challenge due to their multidisciplinary nature $[2,3]$. For most FSI problems analytical solutions of the model equations are impossible to solve and laboratory experiments are limited in scope. With advances in hardware and software technology, numerical simulations and numerical methods become a powerful tool for these problems.

\section{Solution approach}

A coupled (multi field) problem involves two or more interacting fields, for example: a gravity dam with water accumulation, a water tower full of water etc. Such a problem is time dependent and the state of one field is continuously linked to the state of the other field and neither field can be solved independently from the other. Here, the coupling normally occurs through differential equations representing different physical phenomena. The coupled fields can be overlapping, as in the case of seepage and thermo-mechanical problems, or they can be nonoverlapping, as in the fluid-structure interaction problems as discussed in this work. Here, the coupling occurs due to the imposed boundary condition at the interface. The fields may be coupled with all the other participating fields or with only a few of them. The coupling in some problems, like in seepage, may disappear when steady state is reached.

Two main approaches exist for the simulation of fluid-structure interaction problems [1-4]: the Monolithic approach and the Partitioned approach. 
The monolithic approach treats the structure and the fluid in the same mathematical framework and forms a single system equation for the entire problem which is solved simultaneously by a unified algorithm. This approach often achieves better accuracy, but requires more resources and specific knowledge to develop a unique code for every particular problem.

On the other hand, the partitioned approach treats the fluid and the structure as two computational fields which can be solved separately with their respective mesh discretization and numerical algorithm. The partitioned approach preserves software modularity because the existing fluid solver and the existing structural solver are coupled. Moreover, the partitioned approach facilitates the solution of the fluid equations and the structural equations with different, possibly more efficient techniques which have been developed specifically for either the fluid equations or the structural equations.

Thus the partitioned approach has various advantages:

(i) the resulting model is very modular,

(ii) it's easy to make any modifications,

(iii) every modification in one field improves the whole model,

(iv) the programmer/improver can have specific knowledge in (only) a single field.

As a result, an effective partitioned method can solve an FSI problem with very sophisticated fluid and structural physical models. The developer of the FSI simulation has to only unify two different solvers in one task, to achieve an accurate and an efficient FSI solution with minimal code modifications. Special challenges arise when the structure has large displacements so the model has to track the changes in the boundaries, which can often be cumbersome and create errors.

\section{Numerical models}

\subsection{Introduction}

All solutions shown here are based on the partitioned scheme where individual fields are solved independently by considering the interaction information transfer between them at every stage of the solution process. This approach allows the usage of ordinary approaches and appropriate mathematical/physical models for separate fields (the structure and the fluid) with the inclusion of minor modifications for the influence of interactions.

The models and the software developed for the fluid are based on the NavierStokes equation and the SPH method [5-8]. The models and the software developed for the structure are based on the finite elements method (FEM) for the spatial discretization and the finite differences method for the time discretization of the system [9-11].

\subsection{Numerical model for the fluid}

A fluid is a substance (either a liquid or a gas) that continuously deforms under the action of applied surface stresses. Fluid flow may be classified as either 
inviscid or viscous. Inviscid flows are frictionless flows characterized by zero viscosity. No real flows are inviscid, but there are numerous fluids and flow situations in which viscous effects can be neglected. Inviscid flows may be further classified as either compressible or incompressible, depending on whether density variations are large or relatively unimportant. In this study the fluid is considered inviscid and weakly compressible.

\subsubsection{Navier-Stokes equations}

The Navier-Stokes equations are a set of equations that describe the motion of fluid substances such as liquids and gases [5-7].

$$
\rho \frac{\partial v_{i}}{\partial t}=\rho R_{i}-\nabla p+\mu \nabla^{2} v_{i}
$$

In the above equations $v_{i}$ represents the vector of velocity, $\rho$ is the massdensity, $\mathrm{p}$ is the pressure, $\mu$ is the viscosity of the fluid, and $\mathrm{R}_{\mathrm{i}}$ is the sum of external forces acting on the fluid, e.g. gravity. The Navier-Stokes equations are a base point for all fluid movement simulations.

For complex situations, involving cavitation, turbulence, aerodynamics, hydrodynamics and many more, solutions of the Navier-Stokes equations can only be found with the usage of computers.

\subsubsection{SPH method}

The fundamental principle of the SPH method is to approximate any function $A(\vec{r})$ with a unique, uniform, smooth and compact kernel function $W\left(\vec{r}-\vec{r}^{\prime}, h\right)$ that is defined all over the space $\Omega$, with the smoothing length $\mathrm{h}$.

$$
A(\vec{r})=\int_{\Omega} A\left(\vec{r}^{\prime}\right) W\left(\vec{r}-\vec{r}^{\prime}, h\right) d \vec{r}
$$

where $\mathrm{r}$ is any point in $\Omega$. The smoothing length $\mathrm{h}$, or core radius, is a scaling factor that controls the smoothness or roughness of the kernel. The obtained results are critically dependent on the choice of the kernel function (fig. 2).

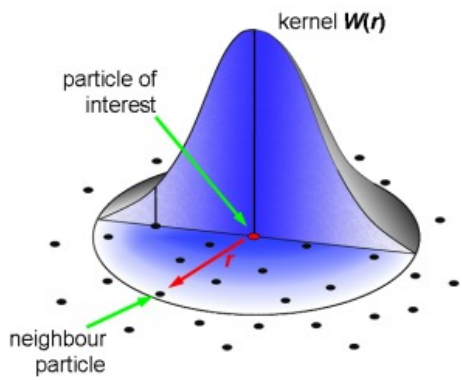

Figure 2: Kernel function.

This approximation, in discrete notation, leads to the approximation of the function $A(\vec{r})$ at a particle of interest: 


$$
A(\vec{r})=\sum_{b} m_{b} \frac{A_{b}}{\rho_{b}} W\left(\vec{r}-\vec{r}^{\prime}, h\right)
$$

where the summation is over all the neighbouring particles within the region of the kernel function. The mass and the density are denoted by $m_{b}$ and $\rho_{b}$ respectively.

The kernel function should satisfy several conditions such as positivity, compact support, and normalization. Also, it has to be monotonically decreasing with increasing distance from the particle and behave like a delta function as the smoothing length tends to zero. One of the often used kernel functions is the Gaussian function [6-8], defined by:

$$
W\left(\vec{r}-\vec{r}^{\prime}, h\right)=\frac{1}{\left(2 \pi h^{2}\right)^{3 / 2}} e^{-\left(r^{2} / 2 h^{2}\right)}
$$

which is also used in this work.

The SPH method assumes that the particle masses and mass-densities for all particles are known before the method starts. The particle mass is a user defined constant, but mass-density is a continuous field of the fluid, which must be computed [6-8].

This is facilitated by the usage of an equation of state to determine fluid pressure. In this work the Tait equation of state is used which defines the relationship between pressure and density according to expression:

$$
P=\frac{c_{0}^{2} \rho_{0}}{\gamma}\left[\left(\frac{\rho}{\rho_{0}}\right)^{7}-1\right]
$$

where $\rho_{0}$ is the reference density (often $\rho_{0}=1000 \mathrm{kgm}^{-3}$ ) and $\mathrm{c}_{0}$ is the speed of sound at reference density.

Furthermore, the fluid has to satisfy the momentum conservation equation:

$$
\frac{\partial \vec{v}}{\partial t}=-\frac{1}{\rho} \nabla P+\vec{g}+\vec{\Theta}
$$

where $g$ is the Earth's acceleration: $\vec{g}=(0,0,9.81)$, and $\vec{\Theta}$ is the diffusion term. In this model the artificial viscosity proposed by Monaghan [6-8] has been used for formulations of the diffusion term.

Changes in the fluid density are calculated with:

$$
\frac{\partial \rho}{\partial t}=\sum_{b} m_{b} \vec{v}_{a b} \nabla W_{a b}
$$

which represents the continuity equation.

The standard Predictor-Corrector scheme is adopted as the solver algorithm [68].

\subsection{Numerical model for the structure}

\subsubsection{Theoretical considerations}

For the dynamic equilibrium of a solid body in motion the principle of virtual work can be used to write the equations independent of material behaviour: 


$$
\int_{\Omega}(\delta \underline{\varepsilon})^{T} \underline{\sigma} d \Omega-\int_{\Omega}(\delta \underline{u})^{T}\left(\mathrm{~b}-\rho_{\mathrm{S}} \underline{\ddot{\mathrm{u}}}-\mu^{\prime} \underline{\dot{u}}\right) d \Omega-\int_{\Gamma_{t}}(\delta \underline{u})^{T} \mathrm{t} d \Gamma=0
$$

where $\delta \underline{u}$ is the vector of virtual displacements, $\delta \underline{\varepsilon}$ is the vector of associated virtual strains, $\mathrm{b}$ is the vector of applied body forces, $\mathrm{t}$ is the vector of surface tractions, $\underline{\sigma}$ is the vector of stresses, $\rho_{\mathrm{S}}$ is the mass density, $\mu^{\prime}$ is the damping parameter and a dot refers to differentiation with respect to time. The domain of interest $\Omega$ has the boundary $\Gamma_{t}$ on which boundary tractions $t$ are specified.

In dynamic analysis, the finite element method can be applied in both analyses: for space and for time. However, it is general practice to use finite elements for space and finite differences for time [9, 10]. This approach is also adopted in this work. Here, the displacement formulation is used because of its simplicity, generality and good numerical properties. For the representation of the finite element, the displacements and the strains and also their virtual counterparts are given by the following relationships:

$$
\begin{aligned}
& \underline{u}=\mathbf{N u} \quad \partial \underline{u}=\mathbf{N} \partial \mathbf{u} \\
& \underline{\varepsilon}=\mathbf{B u} \quad \partial \underline{\varepsilon}=\mathbf{B} \partial \mathbf{u} \\
& \underline{\sigma}=\mathbf{D} \underline{\varepsilon}=\mathbf{D} \mathbf{B} \mathbf{u} \quad \partial \underline{\sigma}=\mathbf{D} \partial \underline{\varepsilon}=\mathbf{D} \mathbf{B} \partial \mathbf{u}
\end{aligned}
$$

where $\mathbf{u}$ is the vector of nodal displacements, $\partial \mathbf{u}$ is the vector of virtual nodal variables, $\mathbf{N}$ is the matrix of global shape functions, $\mathbf{B}$ is the global straindisplacement matrix and $\mathbf{D}$ is the global constitutive matrix.

If (9) are substituted into (8), and if we note that the resulting equation is true for any set of virtual displacements, then the following equation can be obtained in a well-known matrix form:

$$
\mathbf{M}_{s} \ddot{u}_{i}+\mathbf{C}_{s} \dot{u}_{i}+\mathbf{R}_{s}\left(u_{i}\right)=\mathbf{f}_{s}+\mathbf{M}_{s} \ddot{d}
$$

where:

$$
\begin{aligned}
& \left(\mathbf{M}_{s}\right)_{k j}=\int_{\Omega_{s}} \mathbf{N}_{s k}^{T} \rho_{s} \mathbf{N}_{s j} \mathrm{~d} \Omega \\
& \left(\mathbf{C}_{s}\right)_{k j}=\int_{\Omega_{s}} \mathbf{N}_{s k}^{T} \mu^{\prime} \mathbf{N}_{s j} \mathrm{~d} \Omega \\
& \mathbf{R}_{s}\left(u_{i}\right)=\int_{\Omega_{s}} \mathbf{B}^{T}\left(\mathrm{u}_{\mathrm{i}}\right) \sigma_{i} \mathrm{~d} \Omega \\
& \left(\mathbf{f}_{s}\right)_{i}=\int_{\Omega_{s}} \mathbf{N}_{s k}^{T} \mathbf{b}_{i} \mathrm{~d} \Omega+\int_{\Gamma_{t}} \mathbf{N}_{s k}^{T} \mathbf{t}_{i} \mathrm{~d} \Gamma
\end{aligned}
$$

where $\mathbf{N}$ is the matrix of global shape functions [10], $\mathbf{M}_{s}, \mathbf{C}_{s}, \mathbf{R}_{s}\left(u_{i}\right)$ are the wellknown matrices of mass, damping and stiffness, respectively, and $\ddot{d}$ is the ground excitation caused by an earthquake.

For real structures, the strain-deformation relationship is generally non-linear:

$$
\varepsilon=\mathbf{B} \mathrm{u} \quad ; \quad \mathbf{B}=\mathbf{B}(\mathrm{u})
$$


which represents the so called geometrical nonlinearity. In fact, because of geometry transformations, the array $\mathbf{B}$ is not linear but dependent on system displacements. The $\varepsilon$-u relationship is known as the model of geometry.

Structures may undergo either: (i) small deformations which are negligible compared to the dimensions of the body; or (ii) large (or finite) deformations in which the theory of small deformations is no longer valid. The second type of deformations is termed as a geometrically nonlinear problem and special procedure is required for its solutions. In many real civil engineering structures geometric nonlinearity can be neglected.

The relationship between stress $(\sigma)$ and strain $(\varepsilon)$ is also generally nonlinear, and represents material nonlinearity. It is usually called the material model or the constitutive relationship.

All real materials have nonlinear behaviour, but in many cases the material can be consider linear. Many types of material models were developed to represent the variety of behaviours such as linear elastic, nonlinear elastic, elasto-plastic, viscoelastic, visco-plastic, creep, cracking or fracture etc. Different hardening laws such as isotropic and kinematic hardening also developed in the plastic and viscoplastic models. Apart from the linear elastic models, all of these representations are, in some sense, nonlinear.

\subsubsection{Implemented elements}

The presence of shells is common in the engineering practice. This is logical because it's well known that shells are economically efficient structures that have been used for a long time. In engineering practice the term shell refers only to a curved thin structure while other flat structures are called plates which are only a simplified case of shells.

In the developed model thin curved (or flat) structures can be modelled with 8node or 9-node degenerated shell elements, with 6 degrees of freedom in each node (fig. 3).

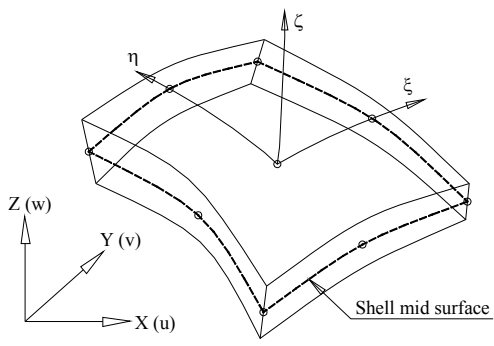

Figure 3: Shell element.

Those elements were primarily developed for the simulation of reinforced concrete structures and/or steel structures, and include the most important nonlinear effects of reinforced concrete behaviour: yielding in compression and opening and propagation of cracks in tension, as well as the nonlinear behaviour of steel (reinforcing or structural) [12]. Also, those shell elements are free of membrane and shear locking, according to [11]. 
For structure modelling of spatial (3D) problems, 20-node and 27-node ("brick") elements are also incorporated in the model. These elements can be used to describe the surrounding soil (not used in this paper).

\subsection{Solution concept for the dynamic fluid-structure interaction problem}

As mentioned before, in the partitioned approach the two developed models/softwares are independent. However, those two softwares have to communicate, and exchange information.

The first step in the numerical analysis is to determine fluid pressures on the structure for the initial state (steady state). In some cases those are the hydrostatic pressures, and in some cases, as shown in the example, initial pressures are equal to zero. The next step is solving equation (10), with one additional member: $\mathbf{f}_{\mathrm{fs}}$, which represents the fluid forces on the structure (13).

$$
\mathbf{M}_{s} \ddot{u}_{i}+\mathbf{C}_{s} \dot{u}_{i}+\mathbf{R}_{s}\left(u_{i}\right)=\mathbf{f}_{s}+\mathbf{M}_{s} \ddot{d}+\mathbf{f}_{f s}
$$

The vector of fluid forces $\mathbf{f}_{\mathrm{fs}}$, can be calculated from (14).

$$
\mathbf{f}_{f s}=-\mathbf{Q} \mathbf{P}
$$

where $\mathbf{P}$ represents the fluid pressures on the structure. The interaction matrix $\mathbf{Q}$ in equation (14) includes only the surface integration and is defined as (fig. 4):

$$
\mathbf{Q}=\int_{\Gamma_{i}} \mathbf{N}^{\mathrm{T}} \vec{n} \mathbf{N} \mathrm{d} \Gamma_{\mathrm{i}}
$$

where $\mathbf{N}$ is the matrix of global shape functions [10]. After determining the displacement of the structure, the calculation domain of the fluid is reconfigured.

For the particles close to the reconfigured border it is necessary to re-determine the density and the pressure with equations (5-7). This procedure is effective only for small deflections of the structure, so the time-step analysis should be set so that displacements are really small (less than one tenth of the initial fluid particle spacing).

The solution scheme is presented in fig. 5 .

\section{Example}

A very simple example, that can describe most of the possibilities of the developed model, is presented. The example is the well-known so called "Dam break" with one flexible boundary. The basic geometry and initial water position is shown in fig. 6 .

The fluid used is pure water with mass density: $\rho_{0}=1000 \mathrm{~kg} / \mathrm{m}^{3}$. Water is considered non-viscous (inviscid) and weakly compressible.

In the presented problem all boundaries are fixed except for the downstream boundary (barrier) which is flexible. This barrier is a steel plate with thickness $t_{\mathrm{s} 1}=12 \mathrm{~mm}$. This steel plate is fixed on three sides (bottom, left and right) and the top side is free. 


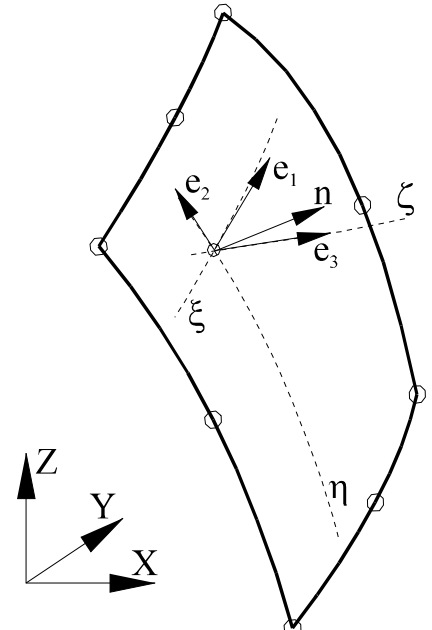

Figure 4: The fluid structure interaction surface and unit norm.

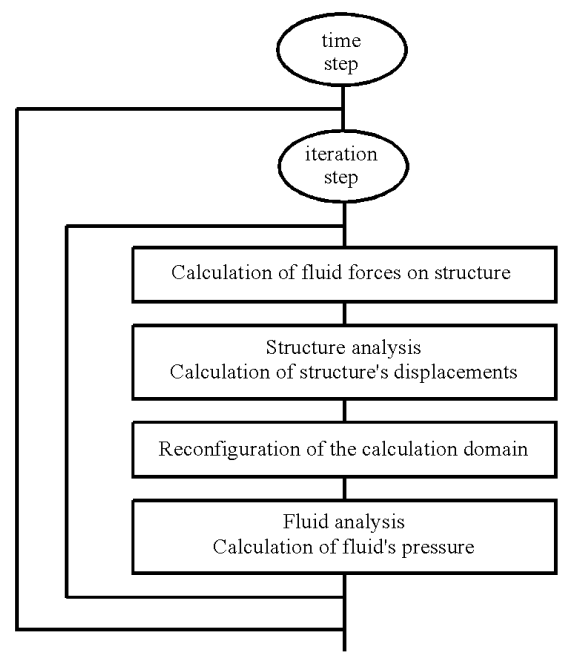

Figure 5: The solution scheme for the fluid-structure coupled problems analysis.
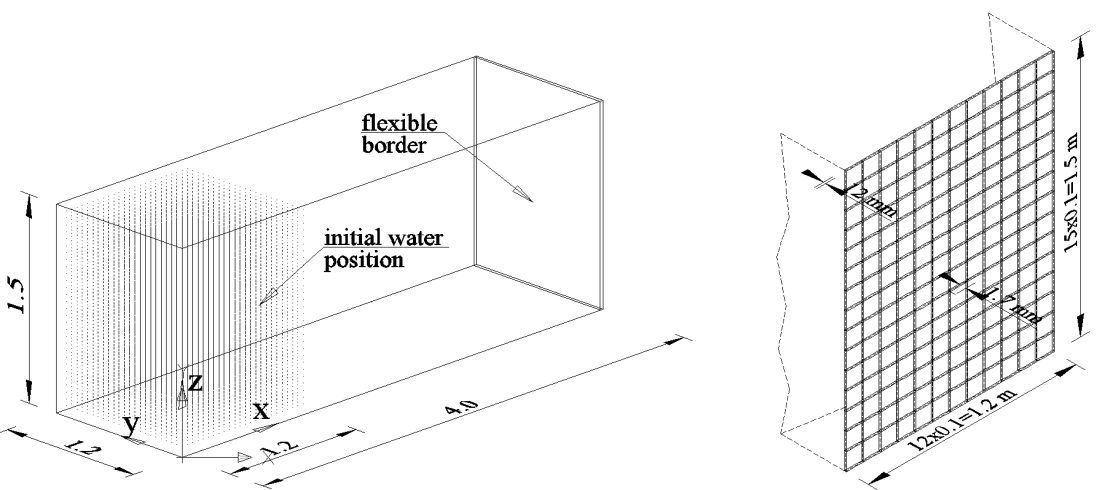

Figure 6: The basic geometry and initial water position.

The other boundaries are considered so called dynamic boundaries. In the SPH method boundary particles are forced to satisfy the same equations as fluid particles (the momentum equation, the continuity equation, the equation of state and the energy equation), but they are fixed - they can't move. Boundary particles are organized in a staggered manner.

The material characteristics used for the steel barrier and water are given in Table 1 where $\mathrm{c}$ is the speed of sound in water and $\sigma_{\text {sy }}$ is the yielding stress of steel. Other parameters are the well-known Young modulus $\left(\mathrm{E}_{\mathrm{s}}\right)$, the Poisson's coefficient $\left(v_{\mathrm{s}}\right)$ and mass densities for water and steel $\left(\rho_{0}\right.$ and $\left.\rho_{\mathrm{s}}\right)$ respectively. 
The particles are initially arranged in a box $1.2 \times 1.2 \times 1.5 \mathrm{~m}$. Total number of particles, including border particles is 48680 , fig. 6 . The time step is $\Delta \mathrm{t}=0.0005 \mathrm{~s}$, and total computational time is $\mathrm{t}_{\mathrm{tot}}=4.0 \mathrm{~s}$.

Table 1: Material characteristics.

\begin{tabular}{|c|l|}
\hline \multicolumn{1}{|c|}{ Fluid - water } & \multicolumn{1}{|c|}{ Structure - steel barrier } \\
\hline & $E_{s}=200.0 \mathrm{GN} / \mathrm{m}^{2}$ \\
$\rho_{0}=1000.0 \mathrm{~kg} / \mathrm{m}^{3}$ & $f_{y} / f_{u}=240.0 / 370.0 \mathrm{MN} / \mathrm{m}^{2}$ \\
$c=1430.0 \mathrm{~m} / \mathrm{s}$ & $v_{s}=0.3$ \\
& $\rho_{s}=7850.0 \mathrm{~kg} / \mathrm{m}^{3}$ \\
\hline
\end{tabular}

The initial pressures on the steel plate (the flexible barrier) are zero, because in the initial state the water didn't touch the barrier. The first contact between the water and the barrier occurred at time $t=0.634 \mathrm{~s}$. The water's influence on the barrier begins at that time.

Figures 7, 8 and 9 show some of the results of the numerical analysis: the movement of water particles, the water pressures on the flexible barrier and the displacements (the deflection) of the barrier in some of the time steps, all visualised in ParaView [13]. The different dot colours represent different pressures. The red dots represent larger pressures then the blue ones.

\section{Conclusion}

The developed numerical model for the Fluid-Structure interaction represents a powerful tool in ascertaining the real behaviour of structures which are in direct contact with and influenced by the fluid. The developed software can simulate the most important nonlinear behaviours of structures, as well as the nonlinear behaviours of the fluid. However, the SPH method itself has a lot of issues that need to be further investigated, especially when the structure has large deformations and impulsive loadings. In such cases the particles usually become highly disordered and results obtained may not always be accurate. Not to mention that large deformations with material nonlinearity are still not a completely solved problem in the structures itself.

One of the main disadvantages of the presented method is its long duration and computer memory consumption. But, with the improvements in processor technology this problem will become secondary. The primary problem still remains the realistic physical and numerical description of reality. 


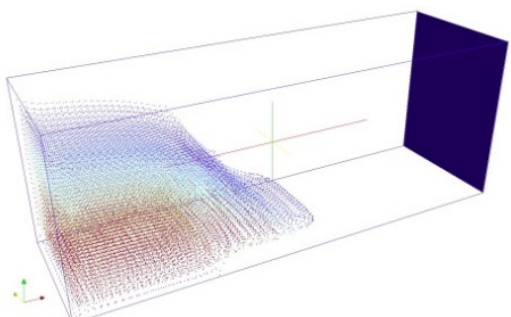

(a) $t=0.35 \mathrm{~s}$

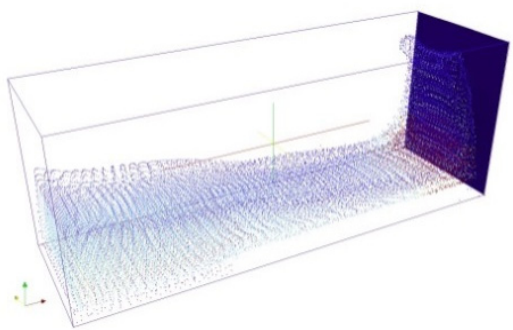

(c) $\mathrm{t}=0.92 \mathrm{~s}$

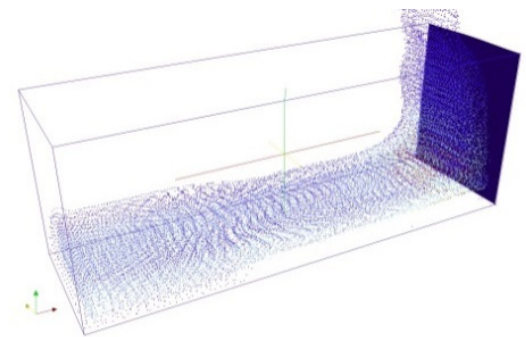

(e) $\mathrm{t}=1.40 \mathrm{~s}$

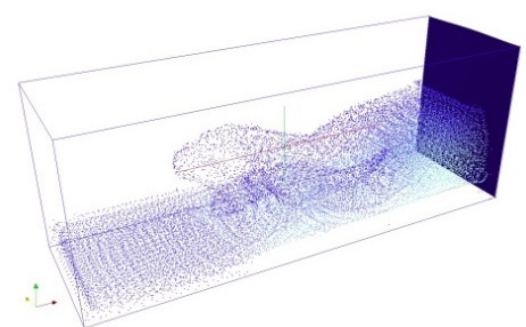

(g) $\mathrm{t}=2.10 \mathrm{~s}$

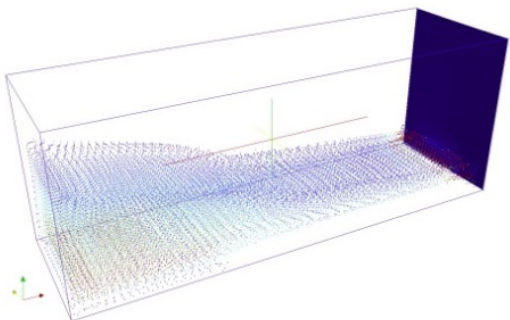

(b) $t=0.70 \mathrm{~s}$

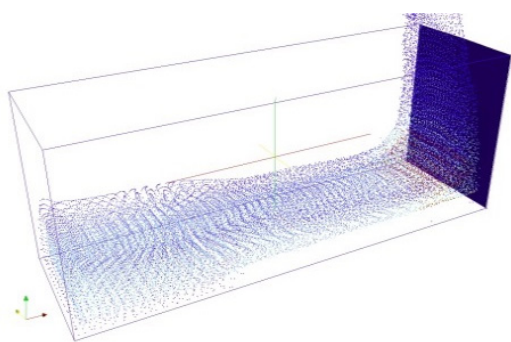

(d) $\mathrm{t}=1.15 \mathrm{~s}$

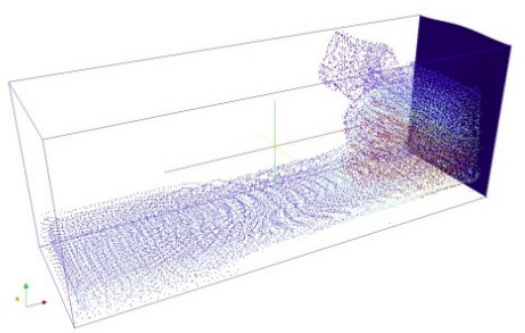

(f) $t=1.75 \mathrm{~s}$

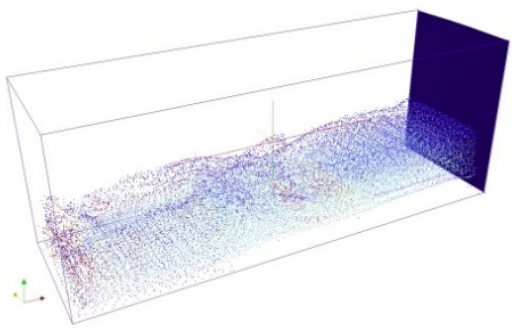

(h) $\mathrm{t}=2.45 \mathrm{~s}$

Figure 7: The movement of water particles and the deflection of the barrier. 


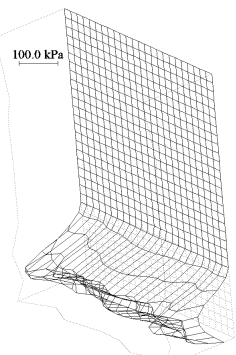

(a) $\mathrm{t}=0.70 \mathrm{~s}$

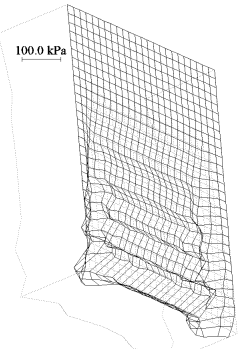

(d) $\mathrm{t}=1.40 \mathrm{~s}$

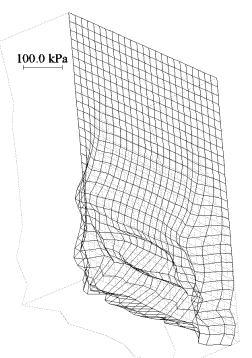

(b) $\mathrm{t}=0.92 \mathrm{~s}$

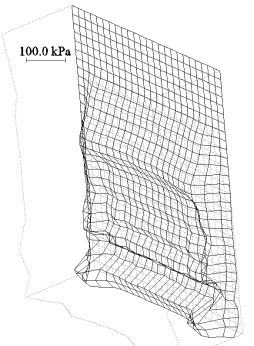

(e) $\mathrm{t}=1.75 \mathrm{~s}$

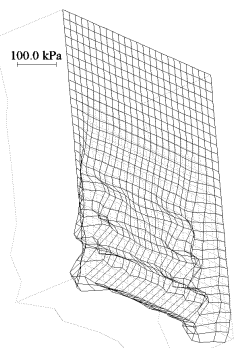

(c) $\mathrm{t}=1.15 \mathrm{~s}$

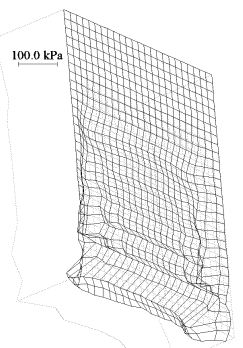

(f) $\mathrm{t}=2.10 \mathrm{~s}$

Figure 8: The water pressures on the flexible steel barrier through time.

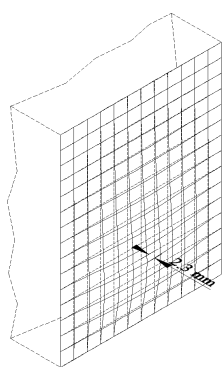

(a) $\mathrm{t}=0.70 \mathrm{~s}$

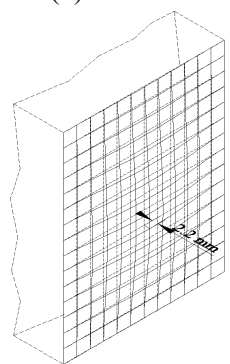

(d) $\mathrm{t}=1.40 \mathrm{~s}$

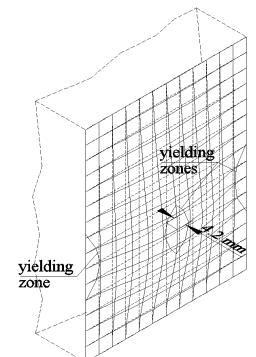

(b) $\mathrm{t}=0.92 \mathrm{~s}$

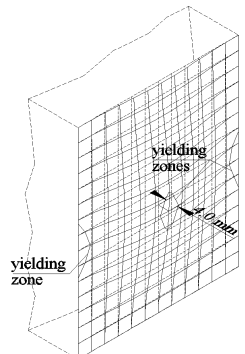

(e) $\mathrm{t}=1.75 \mathrm{~s}$

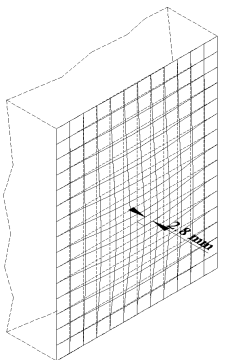

(c) $\mathrm{t}=1.15 \mathrm{~s}$

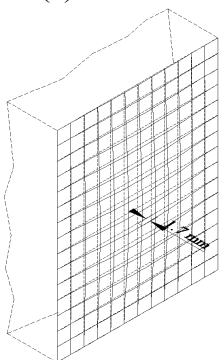

(f) $\mathrm{t}=2.10 \mathrm{~s}$

Figure 9: The deflection of the flexible steel barrier through time (enlarged 20 times). 


\section{References}

[1] Hou, G., Wang, J. \& Layton, A., Numerical Methods for Fluid-Structure Interaction - A Review, Commun. Comput. Phys., 12(2), pp. 337-377, 2012.

[2] Chakrabarti, S. K. (ed.), Numerical Models in Fluid Structure Interaction, Advances in Fluid Mechanics, 42, WIT Press, 2005.

[3] Dowell, E. H. \& Hall, K. C., Modelling of fluid-structure interaction, Annual Review of Fluid Mechanics, 33, pp. 445-490, 2001.

[4] Paul Dilip, K., Efficient dynamic solutions for single and coupled multiple field problems, $\mathrm{PhD}$ Thesis, University College of Swansea, 1982.

[5] Braithwaite, J., An Introduction to Hydrodynamics, Online: https://astro.uni -bonn.de/ _jonathan/misc/hydro_notes.pdf, 2011.

[6] Liu, G.R. \& Liu, M.B., Smoothed Particle Hydrodynamics: A Meshfree Particle Method, World Scientific, 2003.

[7] Kelager, M., Lagrangian Fluid Dynamics Using Smoothed Particle Hydrodynamics, DIKU, University of Copenhagen, 2006.

[8] Gesteira, M.G., Rogers, B.D., Dalrymple, R.A., Crespo, A.J.C. \& Narayanaswamy, M., The User Guide for the SPHysics code, Online: https://wiki.manchester.ac.uk/sphysics/index.php/SPHYSICS_Reference, 2010.

[9] Bathe, K.J. \& Hahn, W.F., On transient analysis of fluid-structure system, Computers and Structures, 10, pp. 383-391, 1979.

[10] Hinton, E. \& Owen, D.R.J., Finite element software for plates and shells, Pineridge Press, Swansea, UK, 1984.

[11] Huang, H.C., Static and Dynamic Analyses of Plates and Shells, SpringerVerlag, Heilderberg, 1989.

[12] Brzović, D., Šunjić, G., Radnić, J. \& Harapin, A., Numerical Model for Fluid-Structure Coupled Problems under Seismic Load, Materials with Complex Behaviour II, Advanced Structured Materials, ed. A. Öchsner et al., Springer in Germany, 16, pp. 175-198, 2012.

[13] ParaView, Online: www.kitware.com; www.paraview.org. 